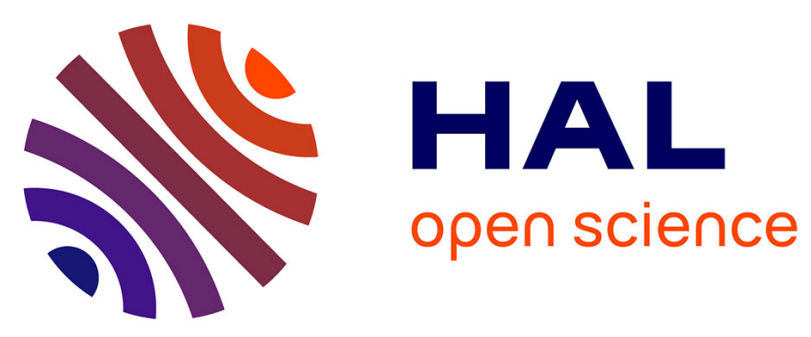

\title{
Les nécroses dues au fluor
}

\author{
Jean Bossavy
}

\section{To cite this version:}

Jean Bossavy. Les nécroses dues au fluor. Revue forestière française, 1965, 12, pp.801-811. 10.4267/2042/24703 . hal-03389844

\section{HAL Id: hal-03389844 https://hal.science/hal-03389844}

Submitted on 21 Oct 2021

HAL is a multi-disciplinary open access archive for the deposit and dissemination of scientific research documents, whether they are published or not. The documents may come from teaching and research institutions in France or abroad, or from public or private research centers.
L'archive ouverte pluridisciplinaire HAL, est destinée au dépôt et à la diffusion de documents scientifiques de niveau recherche, publiés ou non, émanant des établissements d'enseignement et de recherche français ou étrangers, des laboratoires publics ou privés. 


\title{
REVUE FORESTIERE FRANÇAISE
}

Dans ce numéro: J. Bossavy: Les nécroses dues au fluor. - A. Michel: Peut-on escompter les ressources futures d'un boisement. - N. DÉCOURT: Les tables de production pour le Pin sylvestre et le Pin Laricio de Corse en Sologne. - S. PuRcelean: Sur la régénération naturelle du chêne rouvre dans les forêts de feuillus en mélange de la région des collines de Roumanie.

\section{LES NECROSES DUES AU FLUOR}

PAR

\section{J. BOSSAVY}

Ingénieur en Chef du Génie Rural, des Eaux et Forêts à Lyon

\begin{abstract}
Différents articles déjà parus dans cette revue ont présenté aux lecteurs :

A - Une étude générale sur la Vallée de la Maurienne, les résultats des premières observations, ainsi que les dispositifs mis en place dès 1958 en vue d'observer avec plus de précision l'évolution en altitude du périmètre atteint.
\end{abstract}

B - L'extrême sensibilité des 3 essences résineuses les plus couranmment répandues en Europe (sapin pectiné, épicéa, pin sylvestre).

Au fur et à mesure que la documentation reçue devenait plus fournie, plus diversifiée dans ses origines, nous avons pu établir une comparaison entre les différentes échellếs de sensibilité présentées.

Le rapprochement ainsi effectué a montré combien le comportement de ces 3 essences principales se montrait très comparable dans les différentes stations de l'Europe (Allemagne fédérale, Allemagnè de l'Est, Tchécoslovaquie...) en présence des 2 polluants principaux que sont les composés du soufre et ceux du fluor.

Il est en effet apparu que dans toutes les stations et en présence de l'un ou l'autre de ces polluants, les pin sylvestre, épicéa, sapin, étaient en voie de disparition.

Le pin noir d'Autriche montre une résistance plus accusée bien qu'il ne soit pas toujours indemne dans certaines stations. Les aiguilles de mélèze portent régulièrement des traces de nécroses, mais du fait de la chute annuelle des aiguilles, ce résineux ne semble pas subir l'effet cumulatif, très nettement marqué sur les résineux dont les aiguilles se renouvellent avec une périodiçité de plusịieurs années (3 à 7 ans). 
C - Tout dernièrement enfin, nous avons présenté une étude plus détaillée sur le comportement de la flore arbustive et herbacée toujours dans le cas particulier d'une pollution de l'air due au fluor.

Nous voudrions aujourd'hui donner un peu plus de détail et préciser comment se présentent les nécroses sur les tissus foliaires (aiguilles, feuilles) des différentes familles végétales.

Contrairement en effet à ce que l'on pourrait penser, ces atteintes ou brûlures peuvent revêtir un aspect assez différent d'un végétal à l'autre, leur caractère particulier se traduit dans la forme, dans la couleur, et parfois dans le mode de développement.

Le seul point commun à noter, reste cependant que les nécroses débuteront chez les résineux toujours à l'extrémité des aiguilles et se manifesteront sur les feuillus, soit uniquement à l'extrémité lorsqu'il s'agit de feuilles longues èt étroites (glaieul, iris, colchique, prénanthe, pêcher...) soit presque simultanément à l'extrémité de la feuille et sur les bords du limbe lorsqu'il s'agit de feuilles larges et entières (hêtre, gentiane...).

Sur les feuilles profondément dentées ou lobées, les premiers signes de la nécrose seront apparents à l'extrémité des dents ou des lobes (aubépine). Souvent la partie nécrosée prend tout au début de l'attaque une forme en $\mathrm{V}$ dont la pointe est dirigée vers le pétiole, mais très rapidement la nécrose se développera le long du limbe, la plage atteinte prend alors une forme très différente.

Il est courant de constater une légère décoloration de la chlorophylle par petites plages internervales de couleur jaunâtre disposées aussi bien le long du limbe qu'à l'intérieur même de la feuille (hêtre, châtaignier).

Chez certains végétaux, le cerisier par exemple, les parties nécrosées se détacheront très facilement en suivant une ligne ondulée.

La couleur de ces nécroses présente d'autre part, une gamme très étendue; elles seront tantôt noirâtre (noyer), tantôt beige plus ou moins foncé, brune ou rouille, pâle et presque transparente, séparée souvent de la partie restée verte par un liseré plus foncé bien individualisé; parfois au contraire, il n'y aura aucune ligne séparative et la transition sera brutale et nettement tranchée entre partie nécrosée et partie saine.

Dans certains cas, seules les feuilles de l'extrémité du rameau porteront des nécroses plus ou moins développées, ce qui est très certainement en relation avec le stade de développement de chaque élément, avec le temps (sec ou humide, nuageux ou non), la direction des vents.

Dans d'autres cas, et certains résineux en sont un exemple typique (sapin, pin sylvestre), toutes les aiguilles de même âge insérées sur un rameaụ donné porțeront avec une remarquable régu- 
larité des nécroses d'une couleur, d'une forme et d'un stade de développement parfaitement identiques.

Les manifestations apparentes sont donc très diverses, afin de mieux éclairer ceux qui ont à se préoccuper de ces problèmes, nous allons examiner successivement quelques cas particuliers et présenter pour chacun d'eux les caractères les plus marquants (1).

\section{I - Feuillus.}

\section{I-1. - ARbres FrUItiers.}

CERISIER (Cerasus). - La nécrose présente une teinte brun rougeâtre, la feuille souvent gaufrée prend une couleur vert métallique; la partie nécrosée se détache fréquemment, la découpe se produisant en suivant une ligne sinueuse.

Chataignier (Castanea). - Les premiers symptômes se produiront, soit à l'extrémité des petites dents de la feuille, soit sur le bord du limbe dans le sinus séparant les dents, la nécrose apparait de couleur beige clair. Son développement ultérieur se fera, soit de façon discontinue, soit de façon continue le long du limbe, jusqu'à constituer une large plage marginale résultat de la soudure des petites nécroses initiales.

Luzerne (Medicago). - Les folioles sont marquées d'une nécrose apicale brun clair en forme de $\mathrm{V}$ ouvert vers la pointe, mais comme chez la plupart des feuillus, tous les éléments foliacés ne sont pas marqués avec la même régularité.

Noyer (Juglans). - La nécrose apicale présente une teinte brun foncé presque noire, en forme de $\mathrm{V}$, mais le développement rapide des brûlures marginales donnera une forme de $V$ largement ouvert vers le pétiole (disposition exactement opposée).

Un certain zonage apparait souvent sur le bord des parties nécrosées.

PÊcher (Persica). - Seule la pointe sera touchée, la tache brune plus ou moins claire se développera peu, car la feuille tombera très rapidement.

PoIrier (Pirus). - La nécrose de couleur noire gagne rapidement chez certaines variétés l'ensemble du limbe ne laissant subsister qu'une plage verte étroite de part et d'autre de la nervure centrale, chez d'autres variétés la nécrose se maintiendra apicale.

SaInforn (Hedysarum). - Les extrémités de certaines folioles seulement présentent une partie nécrosée de développement encore réduit à cette date et de couleur brun clair.

(1) Voir les différentes planches donnant pour quelques végétaux l'aspect des nécroses, à la date des observations, soit début juillet 1965. 
VigNe (Vitis). - Les premières atteintes apparaitront de couleur gris verdâtre et la zone brûlée restera souple, ultérieurement une teinte brune tirant plus ou moins sur le rouge se développera en formant souvent des lignes concentriques très distinctes et de quelques mm d'épaisseur.

La vigne a été l'objet des premières observations faites à partir de 1906-1907 en Maurienne par M. le Professeur Holland, à titre d'expert.

Deux albums, l'un de photos, l'autre de dessins à la plume comportant en outre quelques planches d'herbier, ont été très aimablement mis à notre disposition par M. ConTE, avocat à Saint-Jean-deMaurienne, qui a bien voulu également nous autoriser à prendre des photographies des pages les plus caractéristiques de ces albums.

Les quelques végétaux en herbier (vigne, luzerne...) se sont conservés de façon remarquable puisqu'après 50 ans de mise en collection de ces échantillons la séparation reste très nette entre les parties nécrosées et les parties saines.

Nous tenons à adresser nos très vifs remerciements à $M$. ConTE pour son extrême amabilité.

Il nous a été ainsi possible de remonter 50 ans en arrière et de constater que le périmètre atteint s'est très largement étendu depuis le début du siècle et que, d'autre part, les nécroses sur les différents végétaux se développent très fidèlement et très exactement de la même façon en 1965 qu'en 1906. Le fait nouveau à relever reste cependant les atteintes sérieuses portées aux massifs résineux depuis 7 à 8 ans et l'évolution inquiétante que traduit l'observation annuelle de ces forêts de sapin, d'épicéa et de mélèze.

\section{I-2. - ESSENCES FORESTIÈRES.}

Tilleul (Tilia). - La bractée de l'inflorescence portera toujours les premières brûlures sous forme d'une tache brune qui affecte l'extrémité libre de la bractée, les feuilles ne seront touchées que plus tard au cours de la saison.

Hêtre (Fagus). - Les nécroses apparaittront simultanément à la pointe et sur les bords du limbe, celui-ci prend un aspect gaufré sur les bords lorsque les nécroses apparaissent à un stade de développement précédant le complet développement de la feuille.

Des plages jaunâtres traduisant une décoloration de la chlorophylle apparaissent très tôt entre les nervures sur les bords comme sur l'ensemble du limbe.

\section{I-3. - VÉGÉtATION ARBUStive et HeRbacÉe.}

AubÉpine (Crataegus). - La nécrose de couleur brun foncé apparaîtra très nettement à l'extrémité des lobes de la feuille. 
Colchique (Colchicum). - La pointe de la feuille tachée de brun très foncé apparaitra très nettement séparée de la base encore verte par un liseré jaune bien marqué de 3 à $5 \mathrm{~mm}$ de largeur.

EPINe vinetTe (Berberis). - Une des plantes les plus sensibles développera soit à l'extrémité arrondie, soit sur les bords du limbe, des taches brunes bien marquées.

Gentiane (Gentiana). - La nécrose de couleur beige très clair se développe rapidement à partir de la pointe et le long du limbe.

Millepertuis (Hypericum). - Les atteintes apparaitront d'une couleur rouille tirant sur le rouge qui gagne rapidement la moitié ou la presque totalité de la feuille. Cette couleur rouille est très caractéristique.

Myrtille (Vaccinium). - Le plus, souvent marginales, d'une teinte brun assez foncé ces nécroses tranchent très nettement sur la couleur verte du feuillage.

Petit Muguet (Maïanthemum). - Les parties atteintes apparaissent claires presque transparentes, la feuille est rapidement touchée sur plus de la moitié de sa surface, il ne semble pas cependant que sa chute en soit accélérée.

Prenanthe (Prenanthes). - La nécrose se développera lentement et apparait de couleur beige clair sur $2-3 \mathrm{~cm}$ à la pointe des feuilles de l'extrémité de la tige.

Rumex (Rumex). - Les brûlures apparaitront d'une teinte brun assez foncé tirant sur le rouge.

Sceau de Salomon (Polygonatum). - Chez le P. "Odoratum ", la pointe brûlée très tôt en saison, présente un aspect transparent et reste souple, une lisière violacée de 2 à $3 \mathrm{~mm}$ de large de forme irrégulièrement ondulée apparait en bordure de la zone nécrosée, sans être obligatoirement à la limite même de celle-ci. Cette lisière existe également dans la variété " verticillatum », mais elle offre alors une couleur d'un vert bleu nettement tranché.

Silene (Silene). - L'extrémité des bractées prendra une teinte grisâtre souvent veinée de noir.

VeRATRE (Veratrum). - Ce compagnon de la gentiane présentera une nécrose typique de l'extrémité de la feuille de couleur brun foncé, qui s'étendra progressivement en se développant régulièrement d'un bord à l'autre du limbe.

\section{II - Résineux.}

Pin sylvestre.

La pointe brûlée des vieilles aiguilles prend une couleur brun rouge, les aiguilles de l'année restent très vertes au cours des pre- 
miers mois de végétation. La nécrose gagne progressivement le bas de l'aiguille sans qu'apparaisse de zone de transition entre la partie desséchée et la partie encore vivante.

\section{SAPIN PECTINÉ.}

Les vieilles aiguilles et notamment celles âgées d'un an apparaissent touchées avec une régularité très caractéristique. Dès la fin de l'hiver des nécroses d'un brun clair sont développées sur 3 à $5 \mathrm{~mm}$ à l'extrémité de toutes les aiguilles d'un rameau, on note en outre la présence d'un liseré brun foncé de 1 à $2 \mathrm{~mm}$ de largeur séparant nettement la partie brûlée de la partie saine.

Les jeunes aiguilles de l'année conservent après 2 mois de végétation leur couleur verte caractéristique et aucune atteinte n'est alors visible.

Il convient cependant d'éviter une confusion entre les nécroses dues au fluor et les dessèchements provoqués par les pucerons ou les rouilles. Dans ces deux derniers cas, la nécrose apparaît sans doute à l'extrémité des aiguilles, mais elle prend un aspect transparent ou gris clair qui affecte quelques aiguilles seulement d'un même rameau, d'autre part ces dépérissements se développeront rapidement sur toute la longueur ou sur plus de la moitié de l'aiguille.

Un examen à la loupe permet en outre de déceler facilement la présence de l'élément nuisible, puceron, ailé ou non, fixé le plus souvent à proximité du point d'insertion de l'aiguille sur le rameau.

\section{EPICÉA.}

La nécrose ne présente pas une différenciation aussi marquée que chez le sapin, elle apparaîtra d'un brun plus ou moins foncé prenant naissance à la pointe sans qu'apparaisse de zone intermédiaire faisant transition entre la partie saine et la partie brûlée. Une plus grande irrégularité peut en outre être observée, les atteintes ne sont pas toujours apparentes sur toutes les aiguilles du rameau, ceci est surtout très net au stade des aiguilles de jeunesse sur les jeunes plants en pépinière.

Comme pour le sapin, les jeunes pousses resteront d'une couleur verte tant que l'effet cumulatif n'aura pas atteint le seuil de toxicité. Les aiguilles cependant seront souvent d'un développement en longueur réduit par rapport à la normale. Sur un rameau atteint, de nombreuses aiguilles tomberont prématurément et ne subsisteront que celles peu ou pas nécrosées. On pourrait, semble-t-il, rapprocher cette observation du test de l'insolation de Hartig.

Cet auteur considère que deux branches d'épicéa, l'une provenant d'un arbre sain, l'autre d'un sujet d'une station soumise aux pollutions, mises au soleil, la seconde perd ses aiguilles en quelques jours, la première en quelques semaines. 


\section{MÉLÈzE.}

Dès la reprise annuelle de la végétation, les jeunes aiguilles subiront des atteintes. Il a été en outre observé à plusieurs reprises, dans un périmètre restreint $(2-3 \mathrm{~km})$ sous le vent de l'usine, la chute des premières aiguilles trop fortement brûlées et encore peu développées.

De nouvelles pousses apparaissaient alors au cours des semaines suivantes.

Ce phénomène peut ne pas être toujours généralisé et pourrait se révéler en relation avec la marche de l'usine et correspondre notamment au démarrage de certaines cuves au printemps au moment où l'énergie électrique d'origine hydraulique est plus facilement disponible, quoi qu'il en soit les nécroses de couleur beige clair apparaitront à la pointe des aiguilles, celles-ci se courberont et prendront un aspect frisé.

Pour un observateur attentif et averti, les manifestations du froid ne sauraient être confondues avec les atteintes dues au fluor.

Les gelées printanières, en effet, entraîneront un dépérissement de l'ensemble des jeunes pousses de l'année, parfois même des seules pousses latérales comme chez le sapin, alors que chez les feuillus un des caractères propres aux nécroses dues au fluor est l'irrégularité des atteintes à l'ensemble des feuilles d'un même âge insérées côte à côte sur un rameau. D'autre part, l'observation de l'ensemble de la végétation arbustive et herbacée permettra de mieux asseoir le diagnostic.

Les observations resteront de préférence limitées à la période de 2 mois qui suit le départ annuel de la végétation, mais il conviendra d'être très attentif, de faire porter les examens sur les rameaux de différents âges ainsi que sur l'ensemble de la végétation, de noter avec le plus de précision possible l'aspect, la forme, la couleur des parties brûlées.

Les manifestations du froid, de la sécheresse, les attaques des insectes ou des champignons présenteront en réalité des aspects légèrement différents des atteintes dues au fluor; il faut cependant noter que chez les végétaux cultivés (vigne, céréales...) certaines attaques d'insectes et certaines déficiences minérales (magnésium) ou en oligoéléments peuvent entraîner des nécroses rappelant, pour un observateur non averti, celles dues à une pollution de l'air.

Dans ce cas particulier, il ne sera sans doute pas toujours possible d'établir un diagnostic précis, l'analyse des tissus foliaires permettra alors de confirmer ou d'infirmer les premières conclusions tirées de la seule observation visuelle. 

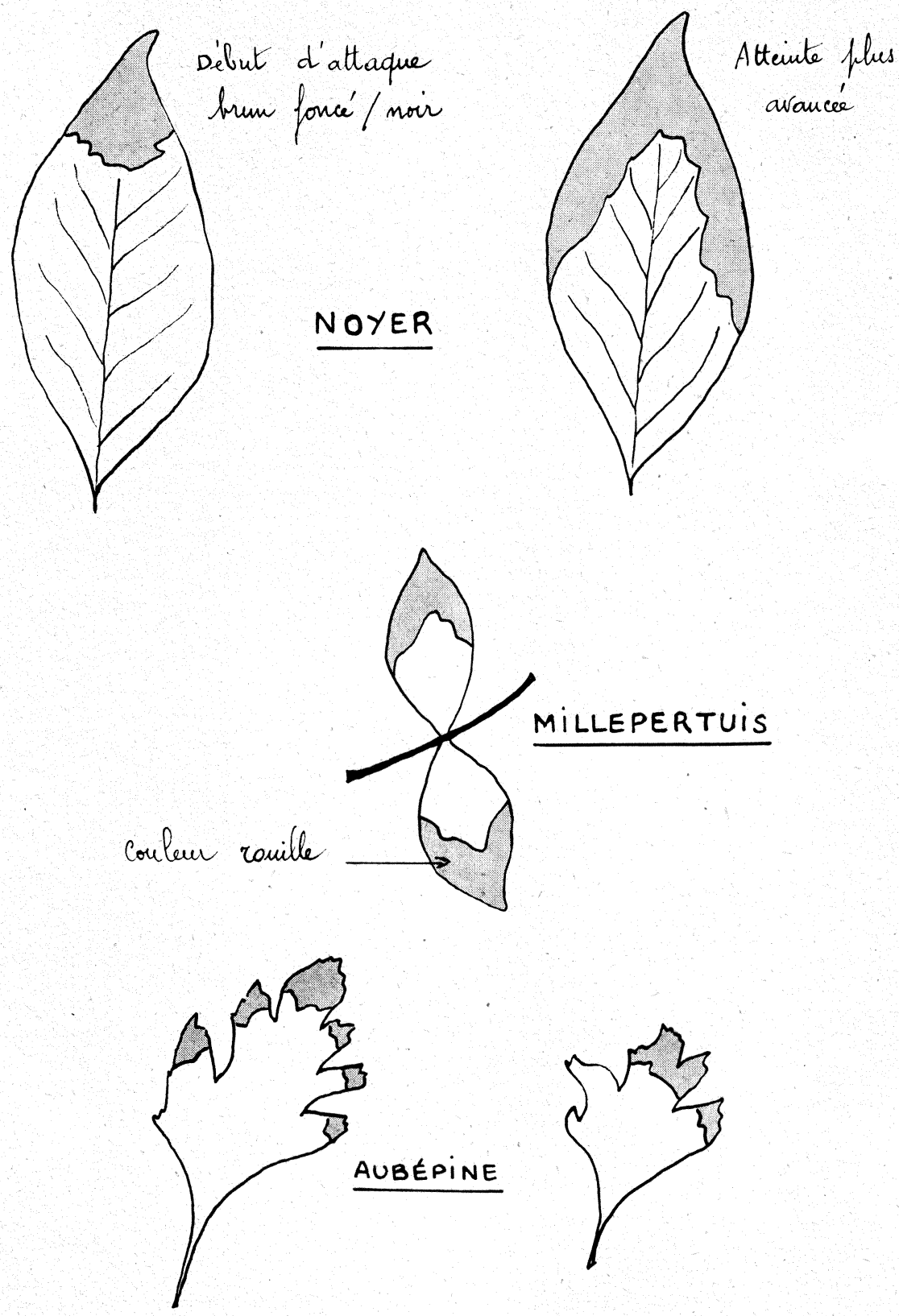

Reproduction d'échantillons cueillis en juillet 1965 et mis en herbier en août.

M. T., août 1965. 
LES NÉCROSES DUES AU FLUOR

809

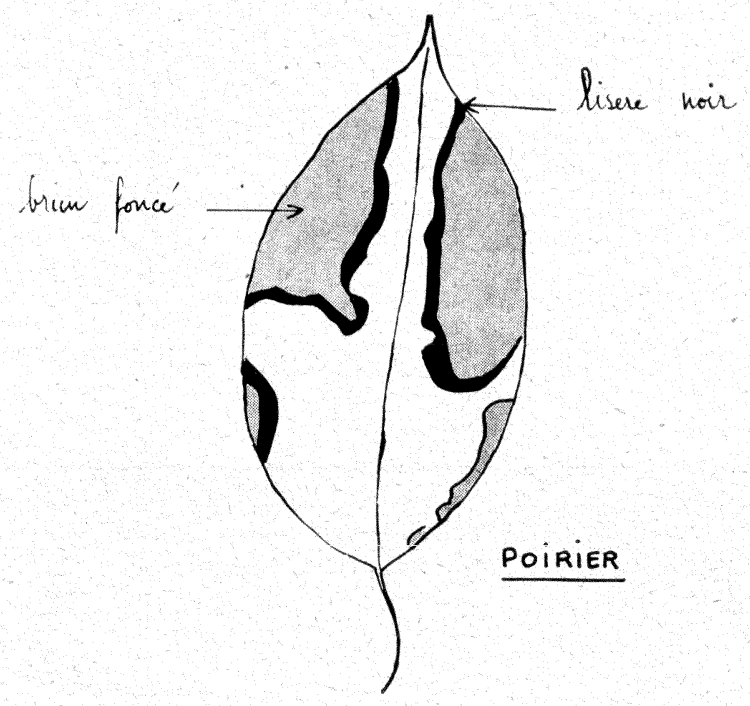

Partic Hécrosé

detachee de la Senille

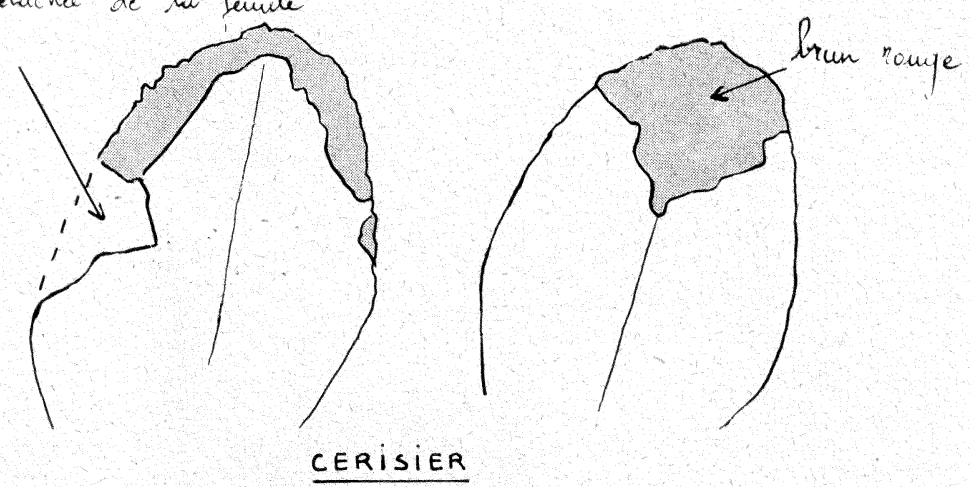




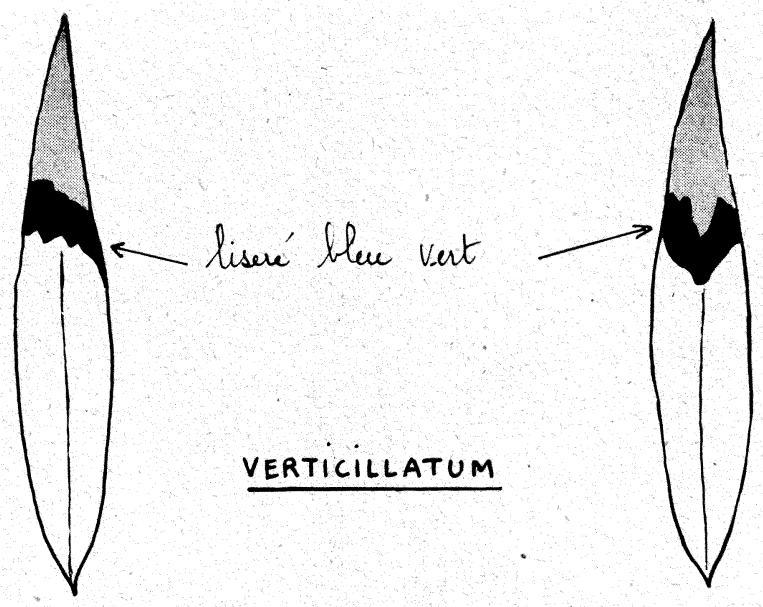

SCEAU de SALOMON
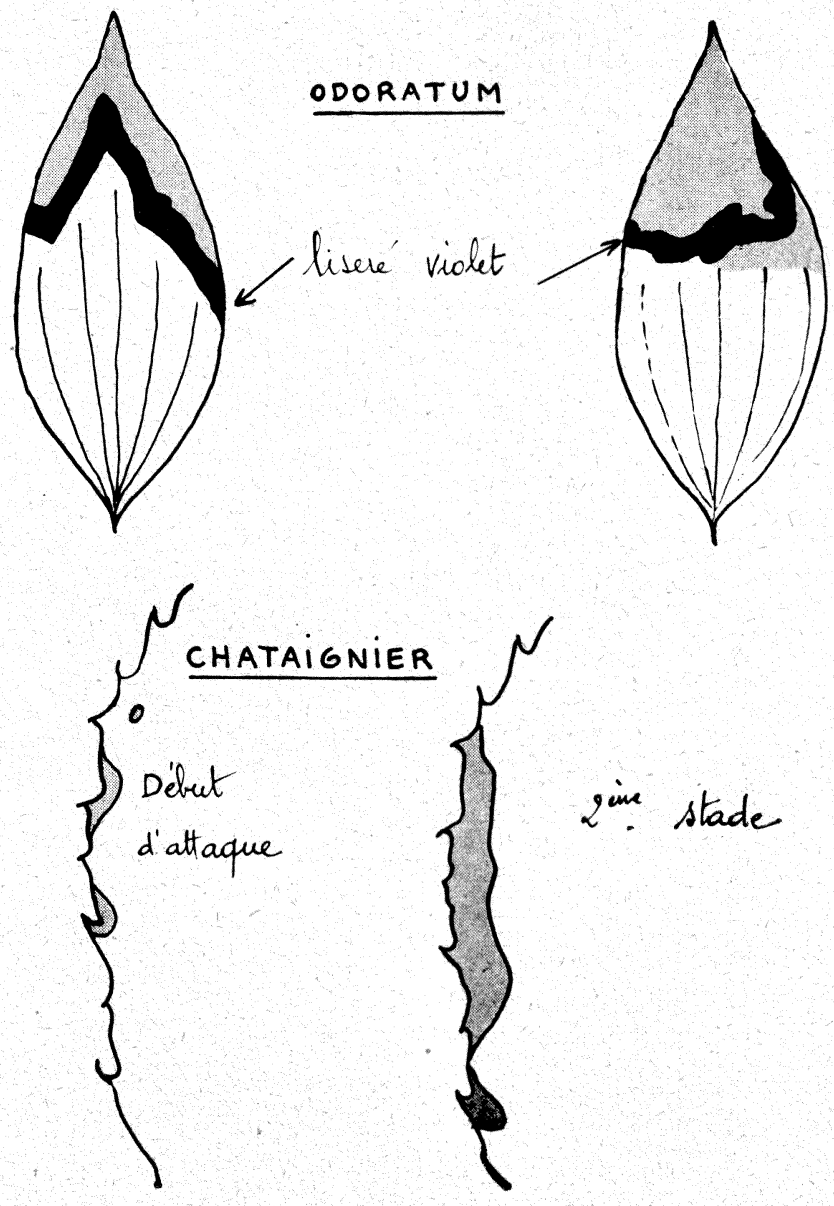

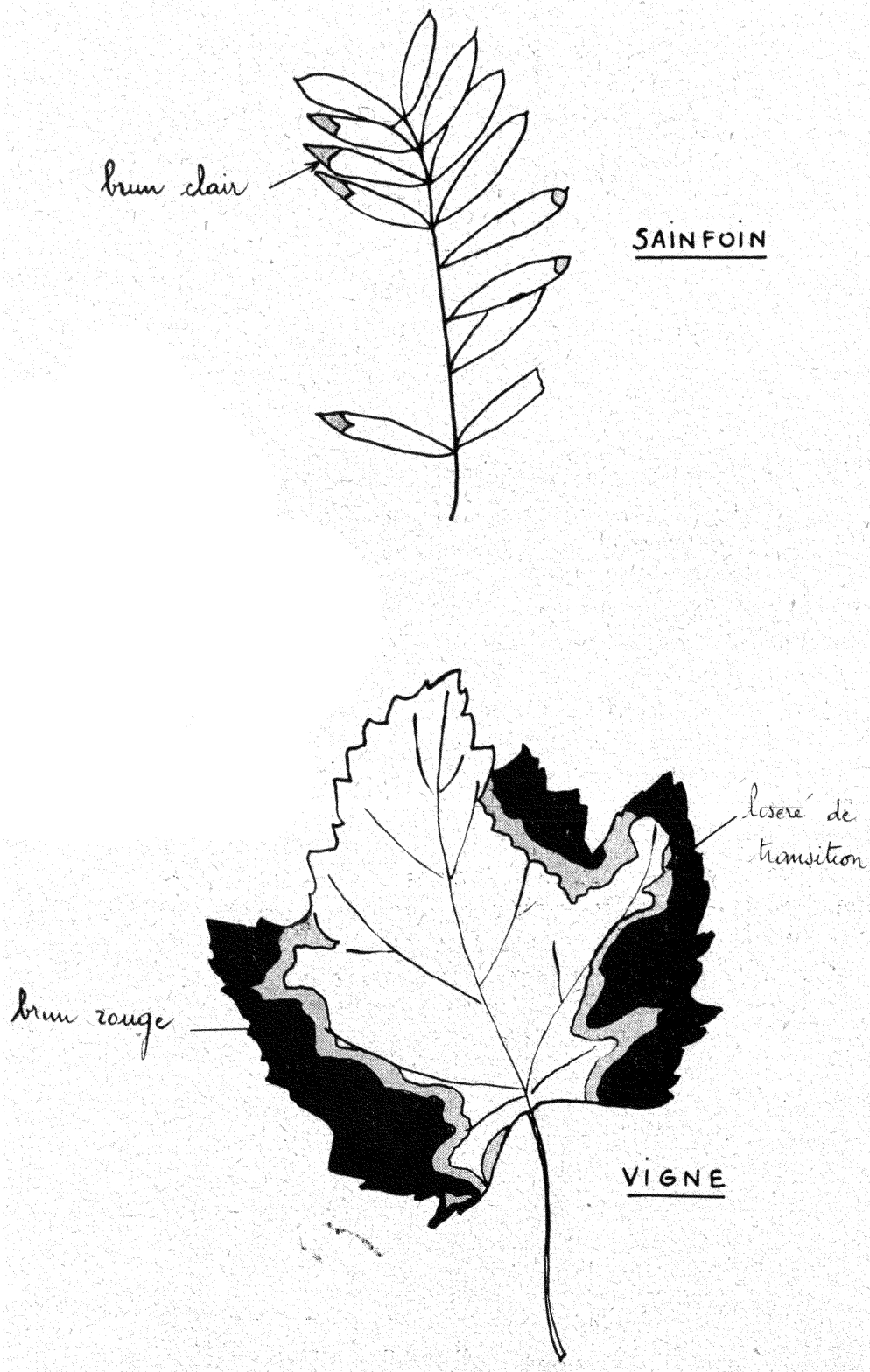